\title{
Evaluating risk reduction by telematic early warning in dam flood scenarios
}

\author{
J. C. P. Palma \& C. A. O. Costa \\ Scientific Instrumentation Centre, \\ Laboratório Nacional de Engenharia Civil, Portugal
}

\begin{abstract}
This paper presents an assessment to the contribution of a telematic Early Warning System (EWS) for reducing the risk of human fatalities due to dam floods. The telematic system consists of a supervisory control post with wireless connection to an array of remote warning units, and involves automation, data communication, acoustic and power supply equipment. The study combines both the modelling of the societal risk associated to flood scenarios and the analysis of functional safety of the telematic system. Both equipment dependability and human factors need to be taken into account in the safety assessment.

Besides the calculation of societal risks, the proposed modelling helps by revealing weak points to consider in the design phase, or to review regularly, as well as the relative importance of different actors and resources involved. A case study shows quantitatively the magnitude of the benefits of a hypothetical telematic EWS in a lightly populated dam valley facing high severity and medium severity scenarios, for typical human action parameters.
\end{abstract}

Keywords: dam failure, dam flood warning, early warning system, probability of failure on demand, societal risk reduction.

\section{Introduction}

There is reported evidence that early warning systems (EWSs) can contribute to the reduction of risk in flood prone valleys, downstream of dams, namely in the case of dam rupture, dam overspill, and of unexpected or exceptionally large discharge operations. The conclusion of a real reduction in life loss by timely issued warnings arises from a considerable list of past dam floods in a wide variety of circumstances regarding dam type, number and location of people at 
risk, relative warning time and flood severity (Graham [1], Ramsbottom et al. [2], Wallingford [3]). The major benefit of an EWS is to increase the mitigation time, i.e. the time left for people in the flood area to self-rescue and, sometimes, even to protect property.

As a consequence, early warning has been adopted in a number of cities and countries, e.g. Myers and Dutson [4], Sutron Corp. [5], MEDD [6], and has also been recommended as part of Emergency Action Planning FEMA [7]. The implementation of a telematic EWS, based on loudspeakers, which is able to broadcast verbal warning messages and conventional siren sounds, has already started in some Portuguese dams (Viseu et al. [8], Costa et al. [9]).

The real effect of an EWS in risk reduction depends on the risk scenarios that are foreseen, their probabilities and the types of undesired consequences to avoid. Economical risks are included in some studies (Carsell et al. [10]), for locations where floods rise in several hours and, with timely issued warnings to the population and emergency services, such that people may protect themselves as well as defend property and move goods to outside the risk area.

The present work is focused on scenarios covering the risk of losing human lives as a consequence of sudden ("flash") floods or flood waves in self-rescue zones downstream of dams. In such areas, roughly at half an hour of flood travel from the dam site, it is assumed that there is no time available to save personal belongings or property. The risk consequences are thus evaluated strictly in terms of the number of possible human fatalities.

\section{A first look at the flood timeline}

Warning signals should be issued soon and clearly, and reach as many people as possible within the area at risk. The time available for the people to self-rescue, called mitigation time, $T_{\mathrm{m}}$, depends on how early the warning is received and understood by the affected persons until they take the decision to leave, as detailed next:

$$
T_{\mathrm{m}}=\max \left(T_{\mathrm{a}}-T_{\mathrm{r}}-T_{\mathrm{w}}-T_{\mathrm{p}}, 0\right),
$$

$T_{\mathrm{m}}=$ mitigation time in a given place, which is the useful time left for people to spend in getting out of the flood risk area;

$T_{\mathrm{a}}=$ time available since a flood is generated until it arrives at that place;

$T_{\mathrm{r}}=$ time spent by dam staff to recognize the situation and decide to warn;

$T_{\mathrm{w}}=$ time spent by dam operators and equipment in issuing the warnings;

$T_{\mathrm{p}}=$ time spent by the people in perceiving the danger and deciding to leave.

The available time, $T_{\mathrm{a}}$, depends on the flood origin and the distance to the place:

in the worst case $T_{\mathrm{a}}$ is reduced to the travelling time of a wave moving downstream the dam to the points of concern (namely in the occurrence of unexpected overspill, dam failure, landslide, sabotage, etc.);

- in other situations the hazard is recognized before the flood passes downstream the dam (e.g. originated by heavy rains, other dam failures in upstream valleys or planned gate operations) and leaves longer periods for warning and evacuating people. 
The response time, $T_{\mathrm{r}}$, depends on the skills of the personnel in charge to recognize the situation and decide to send warnings, as well as on the equipment available for flood imminence detection, on communication resources, on emergency plans and on the collaboration of expert entities (even remotely).

The time spent in issuing warnings, $T_{\mathrm{w}}$, depends on equipment performance and availability, on the promptness of the people in charge, on their expertise in using the EWS central unit for direct warning actions and, in some cases, on the effectiveness of transmitting information for public media broadcast.

People's perception of the danger and decision time to move out, $T_{\mathrm{p}}$, depends on the ability of persons to understand the warnings and to know how to proceed in such cases. Clear predefined messages are very important for increasing public perception; when issued by different media they should preserve coherence. Regular emergency training is another key factor to reduce $T_{\mathrm{p}}$, and should involve as many people as possible of the target group. The proximity interpersonal warning dissemination can also contribute to increase the number of people able to self-evacuate and should be evaluated in training actions. Occasional travellers, which cannot be accounted for by emergency training, may benefit from fixed danger advertisements disseminated in the area and at every road entrance.

The mitigation time available at a given place inside the risk area needs to be higher than the evacuation time, $T_{\mathrm{e}}$, required by an individual to leave that place safely: $T_{\mathrm{m}} \geq T_{\mathrm{e}} . T_{\mathrm{e}}$ depends on the distance that he or she is from a safe location, his/hers physical abilities, time of the day and accessibility conditions. Insufficient mitigation time $\left(T_{\mathrm{m}}<T_{\mathrm{e}}\right)$, either due to late warning or to excessive time spent in perceiving the danger and deciding to run, ultimately leaves the person exposed to the flood risk.

In conclusion, a number of human actions and equipment resources have influence on the warning efficiency with an EWS, i.e. on the proportion of people informed in time and able to self-evacuate relatively to the total number of people in danger. In the following, an attempt is made to quantify the reduction of societal risk that can be expected with the implementation of an EWS in a given situation as well as the relative importance of the major influencing factors.

\section{EWS dependability study}

The architecture considered for the early warning system (EWS) consists of a network of distributed warning units $\left(\mathrm{WU}_{1}\right.$ to $\left.\mathrm{WU}_{n}\right)$ remotely connected to a central system located in a control room (fig. 1). This type of solution has been already adopted in existing EWSs with the authors' specification (Viseu et al. [8], Costa et al. [9]). For the present purposes a description is made of a fictitious dam valley and context.

The control centre is located at a flood safe place. There is a dual (redundant) wireless communication system: by dedicated UHF radio and by commercial GSM. The energy for the central control equipment is provided redundantly by 

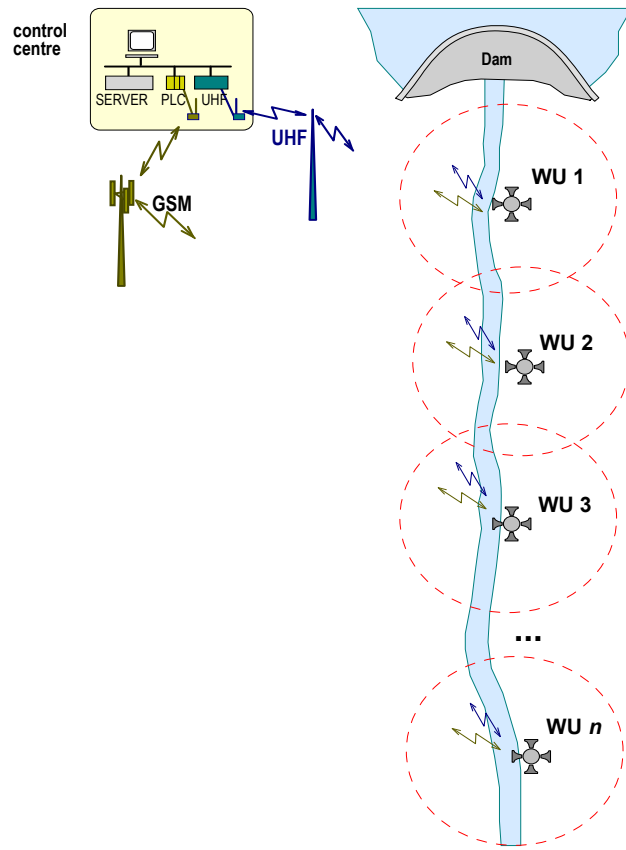

Figure 1: The architecture of the telematic EWS.

the mains supply, through an uninterruptible power supply and even by a portable emergency generator. The supervisory and control PC has improved reliability (disk redundancy and self recovery capabilities). The PC works permanently in monitoring the system state, and is used as human-machine interface, particularly for sending the alarms by the EWS.

Sending messages to all the WU to start the emission of a given type of alarm (and receiving the corresponding acknowledge messages) is quite fast through the dedicated UHF system. If this one fails, automatic switching to the GSM and sending the messages can also be accomplished within a few minutes. Regular diagnostics consists of repeatedly sending test commands and receiving the acknowledgements, via the dual communication system. Emergency commands are sent rarely, on an annual basis, in conjunction with public announcement for training sessions.

Each remote WU has a photovoltaic supply with accumulators able to provide a number of days (5 to 10) at standby autonomy, still keeping energy at the end for an emergency warning session. Each WU includes a local controller, which manages the communications, makes local diagnostic tests and, once ordered by the central system, activates the amplifier coupled to the high speaker system for emitting locally memorized messages and sound warnings.

A simplified model for the dependability analysis of a transmission chain to a single warning unit (WU) can be devised using a reliability block diagram (Smith [11]) as depicted in fig. 2, where the elements are grouped in a small number of main blocks. The failure performance can then be evaluated by 


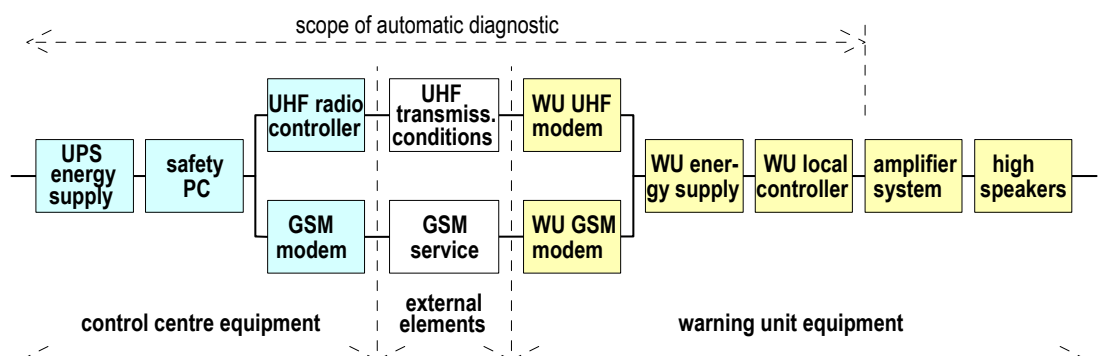

Figure 2: Simplified reliability block diagram of the transmission chain from the control centre to the output of a warning unit.

combinatorial methods for random failures e.g. as suggested in standard IEC 61508 - part 6 [12] for calculating the average probability of dangerous failure on demand $(P F D)$ or even the (un)availability.

All equipment is the object of periodical inspection and maintenance. Most of it is also continuously tested by the central safety PC or by the local controllers. The average probability of dangerous failure on demand of an element can be generically expressed as unavailability by taking into account the period between tests $(T)$, the degree of diagnostic coverage $(C)$, the dangerous failure rate $\left(\lambda_{\mathrm{D}}\right)$ and the mean time to repair $\left(T_{\mathrm{r}}\right)$ :

$$
P F D \approx(1-C) \lambda_{\mathrm{D}}\left(\frac{T}{2}+T_{\mathrm{r}}\right)+C \lambda_{\mathrm{D}} T_{\mathrm{r}} .
$$

All equipment down to the local controller at each WU is continuously monitored, allowing neglecting $T$ comparatively to $T_{\mathrm{r}}$, which yields $P F D \approx \lambda_{\mathrm{D}} T_{\mathrm{r}}$. The average probability of dangerous failure of the central controller and the local controllers are represented by $P F D_{\mathrm{PC}}$ and $P F D_{\mathrm{LC}}$, respectively.

The final elements, i.e. the amplifier and the loudspeakers, are not covered by automatic diagnostic and need periodic full test; their failure metrics $\left(P F D_{\mathrm{AMP}}\right.$ and $P F D_{\mathrm{LS}}$, respectively) are obtained from eqn (2) with $C=0$; full tests with sound emission are only tolerable during emergency training sessions planned with the population, no more than once a year. However, is possible to mitigate this hard condition if, each day, at noon (for example), a single sound, like a clock bell, is broadcasted by each WU.

For evaluating the availability of each power supply $\left(A_{\mathrm{PS}}\right)$, the availability of energy from its source $\left(A_{\mathrm{EN}}\right)$ should be considered in series with a failure block corresponding to the energy processing equipment, with $P F D_{\mathrm{PS}}$ obtained by eqn (2): $A_{\mathrm{PS}}=A_{\mathrm{EN}}\left(1-P F D_{\mathrm{PS}}\right)$. In the photovoltaic case, the availability $A_{\mathrm{EN}}$ combines globally the effect of both the sunny/dark hours and the storage capacity; at the control post $A_{\mathrm{EN}}$ results from the redundancy of the mains and the generator, combined with the UPS storage capacity.

UHF and the GSM alternative communication paths are also characterized by their PFD, $P F D_{\mathrm{UHF}}$ and $P F D_{\mathrm{GSM}}$, respectively, including the contribution of controllers/modems and external resources and conditions. The dedicated UHF radio system is the object of environmental disturbances and field equipment 
unavailability (e.g. poor propagation conditions, failure or lack of energy in relay stations) while the cellular system depends on the lack of continuity of service provided by the mobile phone company.

The overall availability of the communication chain to the population at a given location provided with a WU can be approximately expressed by:

$$
\begin{array}{r}
A_{\mathrm{EWS}} \approx A_{\mathrm{PS} 1}\left(1-P F D_{\mathrm{PC}}\right)\left(1-P F D_{\mathrm{UHF}} P F D_{\mathrm{GSM}}\right) A_{\mathrm{PS} 2} \\
\left(1-P F D_{\mathrm{LC}}\right)\left(1-P F D_{\mathrm{AMP}}\right)\left(1-P F D_{\mathrm{LS}}\right) .
\end{array}
$$

Despite its simplicity, this model puts in evidence the major factors influencing the EWS failure performance, and allows taking important conclusions, provided that sufficiently accurate data concerning availabilities or failure rates are at hand. The values assigned to parameters in this study are as follows: $T=1$ year for all WU equipment; $T_{\mathrm{r}}=1$ week for local controllers and WU power supplies; $T_{\mathrm{r}}=2$ weeks for WU amplifiers and loudspeakers; $A_{\mathrm{PS} 1}=0,9999 ; \quad A_{\mathrm{PS} 2}=0,986 ; \quad P F D_{\mathrm{PC}}=10^{-8} ; \quad P F D_{\mathrm{UHF}}=10^{-2} ; \quad P F D_{\mathrm{GSM}}=5 \times 10^{-2}$; $P F D_{\mathrm{LC}}=10^{-6} ; P F D_{\mathrm{AMP}}=2.65 \times 10^{-3} ; P F D_{\mathrm{LS}}=2.65 \times 10^{-3}$.

$A_{\text {EWS }}$ results are summarized in table 1 as a function of different options for the data transmission media and maintenance intervals.

It is interesting to realize that a redundant solution based on two GSM providers appears almost equivalent to the one involving both dedicated UHF and public GSM. Even recognizing that the former is more likely to suffer from common cause failures the cost of implementation and maintenance of such a system is much smaller than the redundant solution.

Table 1: $\quad$ EWS availability results of the system shown in fig. 2 for different communication options.

\begin{tabular}{|c|c|c|c|c|}
\hline $\begin{array}{c}\text { WU maintenance } \\
\text { interval }\end{array}$ & GSM only & UHF only & $\begin{array}{c}\text { Two GSM } \\
\text { providers }\end{array}$ & $\begin{array}{c}\text { UHF + GSM } \\
\text { redundancy }\end{array}$ \\
\hline$T=1$ year & 0.933 & 0.973 & 0.980 & 0.982 \\
\hline$T=2$ years & 0.926 & 0.965 & 0,973 & 0.975 \\
\hline
\end{tabular}

Table 2: Degrading factors for the EWS availability by decreasing order.

\begin{tabular}{|c|c|c|}
\hline Element or subsystem & Metrics & Value \\
\hline WU power supply & $A_{\mathrm{PS} 2}$ & 0.986 \\
\hline loudspeakers (at WU) & $1-P F D_{\mathrm{LS}}$ & 0.9947 \\
\hline amplifier (at WU) & $1-P F D_{\mathrm{AMP}}$ & 0.9974 \\
\hline communication UHF+GSM & $1-P F D_{\mathrm{UHF}} P F D_{\mathrm{GSM}}$ & 0.9995 \\
\hline CCP power supply & $A_{\mathrm{PS} 1}$ & 0.9999 \\
\hline local controller (at WU) & $1-P F D_{\mathrm{LC}}$ & 0.999999 \\
\hline safety PC & $1-P F D_{\mathrm{PC}}$ & 0.99999999 \\
\hline
\end{tabular}


The most compromising factors for the EWS availability (see table 2) are related to the power supply at each WU, followed by the loudspeakers, the amplifiers and the redundant communication system. The last one becomes prominent in a single GSM or UHF solution.

\section{Calculating the risk with and without the EWS}

In the following an attempt is made to quantify the reduction of risk that can be expected with the implementation of an EWS in a given flood scenario. The factors having greatest relative importance on the results will be identified as well. Firstly, a collective risk evaluation is sought for a general case of nonuniform warning effectiveness and then it is used for risk reduction evaluation.

Let us consider $n$ flood scenarios, $S_{i}, i \in\{1, \ldots, n\}$, affecting the self-rescue zone, where there are $G$ complementary locations numbered $g \in\{1, \ldots, G\}$. Each scenario $S_{i}$ is supposed to occur with frequency $f_{i}$ and, in the case of occurrence, the probability of a person at location $g$ to become a victim is represented by $p_{g \mid i}$.

If the consequences of different flood scenarios on each person are considered statistically independent, then the individual risk (per unit of time) for a person present at location $g$ is obtained by

$$
R_{\mathrm{I}, g}=\sum_{i=1}^{n} f_{i} p_{g \mid i} .
$$

For the evaluation of the collective risk, let $N_{\mathrm{T} g, i}$ be the total number of people at location $g$ exposed to a given hazard scenario $S_{i}$, and $N_{g, i}$ the number of those who did not escape in time. The average number of victims $N_{i}$ per unit of time due to that flood scenario is given by

$$
N_{i}=f_{i} \sum_{g=1}^{G} N_{g, i} p_{g \mid i}
$$

Considering the Bernoulli distribution, with probability $p_{g \mid i}$, for the number of victims at location $g$ in the event of scenario $S_{i}$, the following probabilities of occurrence for each set of exactly $N$ victims are found by

$$
P_{g \mid i}(N)=\frac{N_{g, i} !}{k !\left(N_{g, i}-k\right) !} p_{g \mid i}{ }^{k}\left(1-p_{g \mid i}\right)^{N_{g, i}-k}, \quad 1 \leq N \leq N_{g, i} .
$$

The corresponding cumulative frequencies $F_{g, i}(N)$ of occurring $N$ or more victims at location $g$ due to scenario $S_{i}$ are as follows, given $1 \leq N \leq N_{g, i}$,

$$
F_{g, i}(N)=f_{i} P_{g \mid i}(k \geq N)=f_{i} \sum_{k=N}^{N_{g, i}} \frac{N_{g, i} !}{k !\left(N_{g, i}-k\right) !} p_{g \mid i}{ }^{k}\left(1-p_{g \mid i}\right)^{N_{g, i}-k} \text {. }
$$

The total cumulative frequencies, $F_{i}(N)$, of existing $N$ or more victims over the whole area, due to scenario $S_{i}$, are calculated by taking into account all possible combinations of numbers of victims in different locations making a total number of victims equal or greater than $N$, given $1 \leq N \leq N_{1, i}+\ldots+N_{G, i}$ : 


$$
F_{i}(N)=f_{i} \sum_{\substack{j_{1} \in\left\{1, . ., N_{1, i}\right\} \\ \vdots \\ j_{G} \in\left\{1, . ., N_{G, i}\right\} \\ j_{1}+\ldots+j_{G} \geq N}} \prod_{g \in\{1, . ., G\}} P_{g \mid i}\left(j_{g}\right) .
$$

The number of people occupying the flood prone area and its geographical distribution is a very significant variable for societal risk evaluation. Its determination needs prior knowledge of the area flooded in a given scenario, achieved by hydraulics study. In many cases, it also depends on the season, the day of the week or the time of the day. Further statistical data or assumptions are necessary in order to have representative values.

The number of people remaining in the location when the flood arrives depends on the number of people present before warning, as well as on the effectiveness of warning. Its quantification relies on modelling warning equipment failure and human factors concerning both the operator personnel and the exposed persons. At a location $g$ and a scenario $S_{i}$ there are three quantities to be taken into account independently but with equal importance:

- the probability, $q_{\mathrm{op}}$, of correct operator action both in recognizing the hazard scenario $S_{i}$ and on activating the EWS within a short time lapse;

- the availability, $A_{\mathrm{EWS} g}$, of the EWS at that location; it results from the availability of the control room equipment, the transmission media and the remote WU equipment at the location, as shown in section 3; and

- the probability, $q_{\mathrm{ev} g, i}$, of self-evacuation of a person after a warning.

The average number of people remaining at a location $g$ when the flood arrives, in scenario $S_{i}$, is obtained as follows:

$$
N_{g, i} \approx N_{\mathrm{T} g, i}\left(1-q_{\mathrm{op} i} A_{\mathrm{EWS} g} q_{\mathrm{ev} g, i}\right) .
$$

Round off errors affect the integer value $N_{g, i}$. When numbers are small it is necessary to interpolate between results of eqn (6) with the nearest $N_{g, i}$ values.

Both $q_{\mathrm{op} i}$ and $q_{\mathrm{ev}, i}$ are human error dependent and need to be assessed using appropriate analysis tools (Williams [13], Kirwan [14], Serwy [15]). In terms of human factors, operators' task consists mainly of a timely decision (that is complex, not common, and taken under pressure though with technical background), and then the operation of an equipment set (that is familiar, friendly, with moderate complexity). On the other hand, people's reaction should be considered a complicated, non-routine, stressing task, even being possible to improve it by regular training. Different self-evacuation success probabilities, $q_{\text {ev } g}$, can be used in the model for each location $g$ in order to better represent particular site or population characteristics.

For the present generic purposes, the values to be assigned to these variables were chosen by authors' judgment: $q_{\mathrm{op} i} \approx 0.95$ was kept unchanged for all scenarios and locations, while $q_{\mathrm{ev}, i}$ increases with the distance to the dam. In addition, a uniform value $A_{\mathrm{EWS}} \approx 0.98$ was adopted. All these values need further refinement in order to be valid for a real dam flood site and scenario.

A simplified method is sought for the calculation of $p_{g \mid i}$. Graham [1], Ramsbottom et al. [2], Jonkman et al. [16] provide data and methods for calculating $p_{g \mid i}$, which is mainly a function of depth and velocity of water. 
Following Graham [1] for a high severity flood without warning, or when the warning time is less than 15 minutes, the fatality rate in broad terms is in the order of 0.3 to 1.0 .

In the following, the study will proceed with other assumptions, namely: there are 10 locations in the self-rescue zone and two flood scenarios being considered, a high severity one and a medium severity one. For each scenario $S_{i}$ and location $g$, the population at risk, $N_{\mathrm{Tg}, i}$, the self-evacuation success probability, $q_{\mathrm{ev} g, i}$ and the probability $p_{g \mid i}$ that a remaining person be drawn in that event, are shown in table 3.

Taking into account the data of table 3, the resulting societal risk for each scenario, both with and without warning, are expressed as F-N diagrams shown in fig. 3. The cumulative (or exceedance) frequency axis is normalized by the frequency of occurrence of the underlying scenario $\left(f_{i}\right)$. The value of a global societal risk from all scenarios would be obtained by adding all $\mathrm{F}_{i}-\mathrm{N}$ functions weighted by their corresponding frequencies of occurrence $f_{i}$.

Table 3: $\quad N_{\mathrm{Tg}, i}, q_{\mathrm{ev} g, i}$ and $p_{g \mid i}$ data at each location for two scenarios, assuming uniform values of $q_{\mathrm{op} i}=0.9$ and $A_{\mathrm{EWS} g}=0.98$ in both cases.

\begin{tabular}{|c|c|c|c|c|c|c|}
\hline$i^{\lambda^{g}}$ & 1 & 2 & 3 & 4 & 5 & $6 \leq g \leq 10$ \\
\hline \multirow{3}{*}{1} & $N_{\mathrm{T} 1,1}=3$ & $N_{\mathrm{T} 2,1}=3$ & $N_{\mathrm{T} 3,1}=5$ & $N_{\mathrm{T} 4,1}=5$ & $N_{\mathrm{T} 5,1}=10$ & $N_{\mathrm{T} g, 1}=5$ \\
\hline & $q_{\mathrm{ev} 1,1} \approx 0.3$ & $q_{\mathrm{ev} 2,1} \approx 0.4$ & $q_{\mathrm{ev} 3,1} \approx 0.5$ & $q_{\mathrm{ev} 4,1} \approx 0.6$ & $q_{\mathrm{ev} 5,1} \approx 0.7$ & $q_{\mathrm{ev} g, 1} \approx 0.7$ \\
\hline & $p_{1 \mid 1}=0.75$ & $p_{2 \mid 1}=0.6$ & $p_{3 \mid 1}=0.5$ & $p_{4 \mid 1}=0.4$ & $p_{5 \mid 1}=0.3$ & $p_{g \mid 1}=0.3$ \\
\hline \multirow{3}{*}{2} & $N_{\mathrm{T} 1,2}=1$ & $N_{\mathrm{T} 2,2}=1$ & $N_{\mathrm{T} 3,2}=3$ & $N_{\mathrm{T} 4,2}=3$ & $N_{\mathrm{T} 5,2}=4$ & $N_{\mathrm{T} g, 2}=3$ \\
\hline & $q_{\mathrm{ev} 1,2} \approx 0.4$ & $q_{\mathrm{ev} 2,2} \approx 0.5$ & $q_{\mathrm{ev} 3,2} \approx 0.6$ & $q_{\mathrm{ev} 4,2} \approx 0.7$ & $q_{\text {ev } 5,2} \approx 0.75$ & $q_{\mathrm{ev} g, 2} \approx 0.75$ \\
\hline & $p_{1 \mid 2}=0.3$ & $P_{2 \mid 2}=0.2$ & $p_{3 \mid 2}=0.1$ & $p_{4 \mid 2}=0.1$ & $p_{5 \mid 2}=0.1$ & $p_{g \mid 2}=0.1$ \\
\hline
\end{tabular}

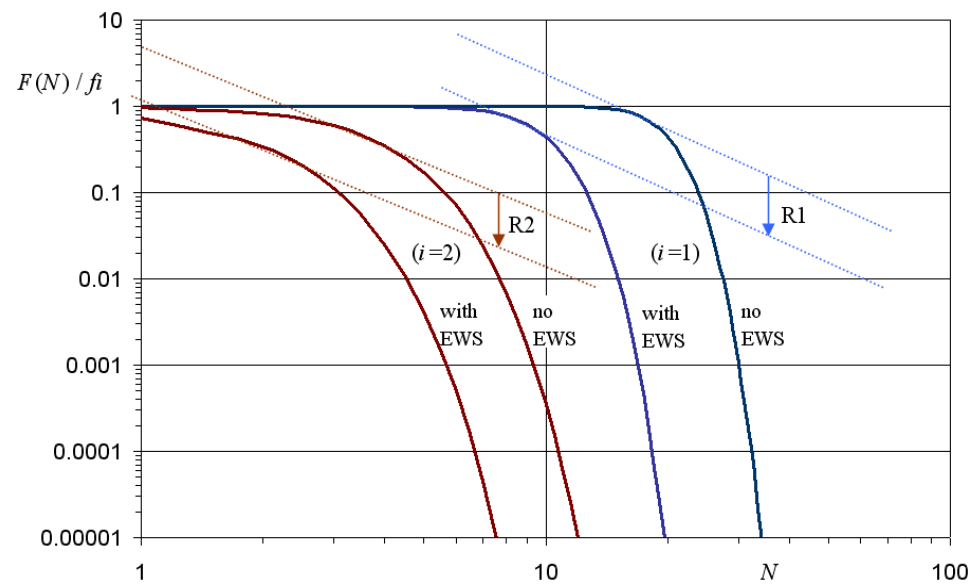

Figure 3: $\quad$ F-N curves representing the societal risk for scenarios 1 and 2, each one with and without the EWS; risk reduction factors $R_{1}$ and $R_{2}$ defined in an index 2 risk aversion metric. 
The relative societal risk reduction obtained from using an EWS can be seen in fig. $3 \mathrm{a}$ as the downward deviation suffered by the $\mathrm{F}_{i}-\mathrm{N}$ curve from the situation without the EWS $\left(A_{\mathrm{EWS}}=0\right)$ to the one with $A_{\mathrm{EWS}}=0.98$.

A reduction factor can be devised as a scalar metric for the risk decrease by measuring the vertical translation suffered by an upper tangent line to the F-N curve, where the slope of this straight line corresponds to a given risk aversion criterion (Vrijling et al. [17]), namely: neutral aversion, and aversion with index 2 (used in fig. 3). Reduction factor values of about 8 , at aversion index 2 , are estimated for using the EWS in the first scenario, and a little less for the second scenario, given the whole set of assumptions that were previously considered.

Any of the three quantities appearing in eqn (9), i.e. $q_{\mathrm{op} i}, A_{\mathrm{EWS} g}$ and $q_{\mathrm{ev} g, i}$, has equal influence on the probability of success. Fig. 4 shows the separated contribution of each one for risk reduction in a scenario of the previous example.

Changing $A_{\text {EWS }}$ from 0 to 1 brings the curve labelled 'without EWS' to the one labelled 'perfect EWS'. Since the EWS has a fairly high availability the later diagram corresponds essentially to the remaining effects of $q_{\mathrm{op} i}$ and $q_{\mathrm{ev}, i}$. Further risk reduction needs the improvement of any of these factors:

- the contribution of highly skilled operators, having all the necessary information support, lowers the curve from 'perfect EWS' to the one labelled 'perfect operator' (by changing $q_{\mathrm{op} i}$ from 0.9 to 1 in the example);

- the improvement of people's response capacity (e.g. by increasing $q_{\mathrm{ev} g, i}$ from 0.7 to 0.8 or higher) could bring the last diagram further down.

In part, the increment of $q_{\mathrm{op} i}$ and $q_{\mathrm{ev} g, i}$ may be achieved by improving operator and public skills in their specific roles, by training, associated with a human error assessment. In addition, factors $q_{\mathrm{op} i}$ and $q_{\mathrm{ev} g, i}$ can also benefit from the investment in operational features of the EWS, namely:

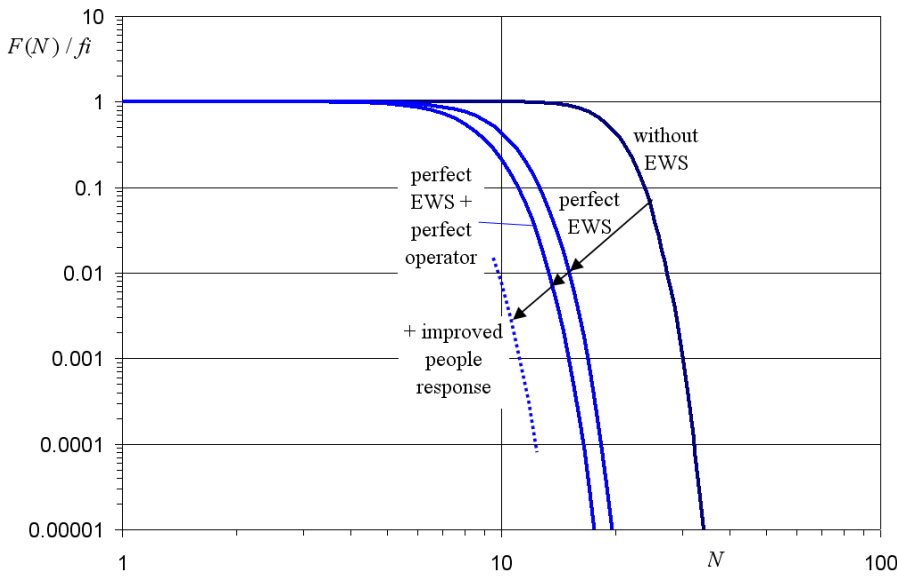

Figure 4: The effect of each factor for the reduction of societal risk. 
- effective coverage of the target people in the whole flood area by the warning facility,

- clearness and comprehensibility of messages or warning signals for the target people at each location,

- human machine interface quality for operators at the control centre.

Formal methods for human error assessment allow to estimate the probabilities $q_{\mathrm{op} i}$ and $q_{\mathrm{ev} g, i}$ in greater detail, for each scenario and each location, respectively. In addition, those methods include a phase of identification of potential human errors (Kirwan [14]), and produce outputs to improve the design of humanmachine interface as well as to define aspects to be addressed in training actions.

\section{Conclusions}

The usefulness of a telematic early warning system (EWS) as a dam flood risk reduction measure was assessed by means of a relatively straightforward method. Major influencing factors were identified and discussed in generic terms. Both qualitative and quantitative results were obtained for a fictitious dam site, with sparse people occupation, in two scenarios corresponding to high severity and medium severity floods. Risk reduction factors of about 7 and 8 were obtained.

These results were based on typical values for the probabilities of correct action by the operators at the control centre and of appropriate public response to warnings.

The effective reduction of risk to be claimed for an EWS is not an intrinsic (fixed) capacity of the system itself but depends also on the performance of human actors, namely, the operators and the target people in the risk area.

In flood risk evaluation there are major contributions of human factors, especially those concerning the ability of operators to recognize the hazard and launch the warnings and the capacity of public reaction after hearing the warnings.

Energy break in remote solar-powered warning units, failures in loudspeakers or amplifiers and communication media outages are expected to be the most detrimental effects for the telematic early warning system under analysis. Correct maintenance, particularly for equipment in the countryside, is essential for preserving the EWS capacity for risk reduction.

The methodology adopted and the results obtained so far were an attempt to proceed towards the evaluation of the risk reduction that can be achieved by using a telematic EWS for dam floods. However, it needs to be the object of discussion, as well as to have some more specific data, before it may be used for assessing real situations, in order to prevent gross over- or under-estimates.

\section{References}

[1] Graham, W., Estimating loss of life due to dam failure, Bureau of Reclamation, publication DSO-99-06, Denver, 1999. 
[2] Ramsbottom, D., Floyd, P. \& Penning-Rowsell, E., Flood Risks to People Phase 1, R\&D Technical Report FD2317, DEFRA/Environment Agency, 2003.

[3] HR Wallingford, Flood Hazard Research Centre - Middlesex University, Risk \& Policy Analysts Ltd., Flood Risks to People - Phase 2, The Flood Risks to People Methodology, R\&D Technical Report FD2321/TR1, DEFRA/Environment Agency, 2006.

[4] Myers, B. \& Dutson, G., Warning System Design When Early Warning is Not Early Enough - Silver Creek Dam and Hosler Dam Case Studies, Proceedings of the 23rd United States Society on Dams Annual Meeting and Conference, pp. 1-17, 2003.

[5] Sutron Corp., Black Rock Dam Early Warning System, Pueblo of Zuni, www.sutron.com/pdfs/Zuni_Dam_EarlyWarning.pdf, 2006.

[6] MEDD, Les ruptures de barräges, Ministère de L'Écologie et du Développement Durable, Dossier d'Information, Risques Technologiques Majeurs, 2004.

[7] FEMA, Federal Guidelines for Dam Safety: Emergency Action Planning for Dam Owners, Interagency Committee on Dam Safety, U. S. Department of Homeland Security, Federal Emergency Management Agency, 1998, reprinted 2004.

[8] Viseu, T., Palma, J. \& Costa, O., Alqueva Dam Early Warning System, Proc. of the 5th International Conference on Dam Engineering, pp. 619626, Lisboa, 2007.

[9] Costa, O., Palma, J., Castro, T. \& Viseu, T., Alqueva-Pedrogão Joint Early Warning System, Proceedings of the International Conference on Large Dams, ICOLD, Brasília, 2009.

[10] Carsell, K., Pingel, N. \& Ford, D., Quantifying the Benefit of a Flood Warning System, Natural Hazards Review, pp. 131-140, ASCE, August 2004.

[11] Smith, D., Reliability, Maintainability and Risk - Practical methods for engineers, 6th edition, Butterworth-Heinemann, 2001.

[12] IEC, International Electrotechnical Commission. International Standard IEC 61508: Functional Safety of Electrical/Electronic/Programmable Electronic Safety Related Systems, Geneva, 2000.

[13] Williams, J. C., HEART - a proposed method for assessing and reducing human error, Proceedings of the 9th Advances in Reliability Technology Symposium, University of Bradford, 1986.

[14] Kirwan, B., The validation of three human reliability quantification techniques - THERP, HEART and JHEDI - part I - Techniques and descriptions, Applied Ergonometrics, 27(6), pp. 359-373, 1996.

[15] Serwy, R., CREAM Calculator, ver. 6, University of Illinois at UrbanaChampaign, http://www.ews.uiuc.edu/ serwy/cream/v0.6beta/, 2009.

[16] Jonkman, S., van Gelder, P. \& Vrijling, J., Loss of life models for sea and river floods. In Wu B et al (eds) Flood Defence 2002, Science Press, 2002.

[17] Vrijling, J., van Hengel, W. \& Houben, R., A framework for risk evaluation, Journal of Hazardous Materials, (43), pp. 245-261, 1995. 